Review Article

Open Access

\title{
Making the Case for Integrated Human-Animal Preventive Health Care System in economically Underdeveloped Communities
}

\author{
Aya Omar ${ }^{1}$ and Rawhi Omar ${ }^{2}$ \\ ${ }^{1}$ Gaines Center for the Humanities Senior Fellow, College of Arts and Sciences-Biology, College of Agriculture- Animal Science, \\ University of Kentucky, Lexington, KY, USA \\ ${ }^{2}$ Professor, Department of Pathology \& Laboratory Medicine, University of Louisville School of Medicine, Louisville, KY, USA
}

\section{Article Info \\ *Corresponding author: \\ Rawhi Omar \\ Professor \\ Department of Pathology \& Laboratory \\ Medicine \\ University of Louisville School of Medicine Louisville, KY, USA \\ E-mail: raomar12@gmail.com}

Received: April 8, 2018

Accepted: April 28, 2018

Published: May 3, 2018

Citation: Omar A, Omar R. Making the Case for Integrated Human-Animal Preventive Health Care System in economically Underdeveloped Communities. Madridge J Vaccines. 2018; 2(1): 44-47.

doi: 10.18689/mjv-1000109

Copyright: $\odot 2018$ The Author(s). This work is licensed under a Creative Commons Attribution 4.0 International License, which permits unrestricted use, distribution, and reproduction in any medium, provided the original work is properly cited.

Published by Madridge Publishers

\begin{abstract}
Health care in economically depressed communities is almost always a hostage to socio-economic "class' systems that typically separate society into a minority of wealthy individuals or groups that are bid against the remaining impoverished majority. That majority, in its struggle for survival, relies heavily on privately owned farm animals for economic sustenance, which, in turn, makes its own health dependent on the health of its animals. An infectious disease that is usually limited to animals (zoonosis) may acquire added virulence so as to inflict the respective animal owners and from their proceeds to inflict the rest of the community regardless of class or wealth. This dynamic is exemplified by the wide spread of rabies, a deadly zoonosis, among people and animals of poor communities in Nepal, whereby the inadequacy of general health provisions, especially preventive anti-rabies vaccination, is at the core of this health crisis. From analyzing that example, it is concluded, that providing adequate preventive healthcare for all, including domestic animals, should not be viewed as an act of charity, but rather as an absolute necessity.
\end{abstract}

Keywords: Health care; Infectious disease; Virulence; Vaccination.

\section{Introduction}

A widespread issue in many impoverished countries is a distinct lack of healthcare infrastructure dedicated to preventing the spread of disease, both zoonotic and otherwise. There are many factors that influence human health in those countries but a great one is the lack of attention placed on zoonoses and the management of disease corridors from animals to humans. "Class" barriers, mistakably, lull the privileged classes to think that infections which may prevail among the poor farmers and their animals will remain limited to those groups and unlikely to reach the clean, well-taken care of, elite. Even in seemingly affluent communities, staunch advocates of absolute capitalism and individual wealth see no guilt in denying universal or preventive health care to those who cannot afford it and view that as a just punishment for "being too lazy" to achieve a higher wealth status and thus deserving what they had coming. Yet, those same rationalizers of class disparities, usually, have no qualms about hiring the "unhealthy poor" as "nannies" looking after their "wealthy" kids or as housekeepers cooking their fancy food. They, apparently, adopt the short-sighted perception that the disease (infection) the nanny or the housekeeper carries is unlikely to cross to their children and to the rest of their household, which is, obviously, erroneous. Not only that it is their children who will get the infection next, but there is a real danger that the infectious 
strains may actually be more virulent than those in the general population. That is simply the case because a poor worker or neighbor who cannot afford preventive vaccinations or other basic health care items and who survives on "leftover", cheap or sometimes "rotten/spoiled" food remnants may end up being immunocompromised and acquire opportunistic infections or diseases that are usually limited to animals (zoonoses) but may jump to immunocompromised humans. Once in a susceptible human host, some of these infections may evolve into more virulent strains or those which developed increased resistance to antibiotics after being undertreated. As more virulent and antibiotic-resistant, such infections will find their way directly to the family of the superior "masters". Those "masters" who understand this dynamic of animal-human interactions should be forthcoming in support of a free or affordable healthcare system that at least guarantees preventive care (vaccination, disease screening, sewage and waste disposal systems, pollution control etc) to the entire community of humans and their animals.

\section{The Impact of Human-Animal Interactions: A Close Look at One Prototype community}

The described human-animal interactions and their respective sequelae are evident in many countries or communities around the world. One of the co-authors on this report (Aya Omar) visited one such community in the country of Nepal and worked closely with affected people and their animals. That community is being reviewed here as a prototype for the impact of poorly managed human-animal interactions on population health and productivity.

The various social and economic issues that have plagued Nepal throughout its recent history culminated in the development of an epidemiologic crisis; specifically, the continual spread of "rabies".

In an economically challenged society, human healthcare is often a luxury and citizens are prone to placing the welfare of the food animals or livestock that provide steady income, over their own. For example, the practice of bathing water buffalo in local water sources is common to many areas of Nepal that lack in health education or feature predominantly agrarian economies. The civil war has also contributed to a large number of health issues with the internal displacement of over 200,000 Nepalese citizens, leaving them without access to basic amenities, let alone proper healthcare. These issues have been further exacerbated by economic and demographic changes, such as new agroindustries without safe waste management, migration, deforestation, encroachment, unplanned urbanization with little to no provisions for safe drinking water, and environmental degradation [1]. Many of these issues directly relate to animal mishandling, such as improper slaughtering and waste removal adding to polluted drinking water systems, while others are contributors to developing corridors for zoonotic disease transfer to other animals and humans.

Historically, Nepal's main problem with the establishment of a widespread healthcare initiative has been lack of education and political will. The prevailing system has heavily favored the wealthy class, extending all the way to privatization of healthcare. Up until 1991, healthcare was mainly present in large cities such as Kathmandu and due to economic strife before and after the civil war; care was limited and restricted mainly to the wealthy upper class. Another large issue is the disparity in education between the rich and the poor. Based on a 1991 census, only 96,977 persons in Nepal attained an education at graduate level and above. Of these only $3.1 \%$ belonged to the lowest caste, even though they made up $20 \%$ of the general population [2]. The national average for literacy rates in 2001 was $53.7 \%$ and $80 \%$ of the population lived in rural areas where the average number of years of school attended was 2.37 [3]. This disparity in education has contributed greatly to the many human health crises plaguing Nepal, but is especially evident when addressing the interactions locals have with animals.

The three main types of parasitic zoonosis in Nepal are cysticercosis, toxoplasmosis, and cystic echinococcosis. However, the zoonotic disease with the highest death toll remains rabies, causing between 10 and 20,000 incidents of infection per year, more than that of all three parasites combined [4]. The spread of rabies is common in many developing countries and post war zone areas due to a lack of vaccination programs, animal control, veterinary care, or human health awareness. It is especially prevalent in Nepal due to many of its unique social, economic and geographic features. Nepal shares an open border with India, an area with the highest incidence of human rabies in the world, where 20,000 deaths occur from the disease per year [5]. Many of the wildlife and street animals infected with rabies cross the border, which contributes greatly to the spread of the disease into regions in southern Nepal. Rabies occurs in two interrelated epidemiologic cycles: an urban cycle involving domesticated and street animals and a sylvatic cycle involving wildlife. The sylvatic cycle has a low impact on the human population and serves more as a vector of transferring the disease to companion and street animals who then bring it to urban areas. Wildlife also has a moderate role in the transfer of rabies to livestock in farms that encroach on wild habitats. The urban cycle is the predominant source of human rabies, as $96 \%$ of all cases between 1991 and 2000 were reportedly caused by dogs. The most recent report states that rabies kills about 100 livestock and 10-100 humans each year. However, 1,000 livestock and 35,000 humans are reported to receive the post-exposure rabies prophylaxis each year [6]. These numbers themselves are likely to be even higher, as many instances of rabies exposure go un-noted.

The development of the animal and human pre- and post- exposure vaccine itself has taken several years to come to fruition and local production of the vaccine remains insufficient, especially in public health facilities where it is needed the most [4]. In these cases, many people are forced to resort to the private market for more expensive vaccines or are denied the post exposure prophylaxis (PEP). The more effective equine or human RIG (rabies immunoglobulin), the formula made from human plasma rather than animal, is 
available only in clinics in Kathmandu and, because of cost, is mainly used by tourists or wealthy citizens.

In rural areas of Nepal is it often difficult and expensive to travel to a city that has the rabies post-exposure prophylaxis. The medicine itself can be expensive and often can't be afforded by lower caste citizens. Some also prefer to seek out traditional remedies and stay home, even though the rabies virus causes fatal encephalitis within two to five days. Additionally, many rural clinics often turn away patients seeking post exposure prophylaxis out of fear of exposure to hospital staff or lack of the proper treatment as there are nation-wide shortages of the medication.

\section{Discussion}

An overarching theme with healthcare in many underdeveloped counties is a focus on symptomatic treatment rather than preventative care [1]. This idea not only refers to the actual medical care people receive, but the premise behind healthcare initiatives as a whole. A relatively common phenomenon in developing countries is to focus all healthcare efforts on immediate threats such as infectious diseases, disaster relief, acute illness, etc. and later follow suit with preventative care. Unfortunately, even those countries that set up successful programs for preventative care, such as rural clinics and non-emergency medical centers, do not function to treat the overall, environmental health of a person. Preventative care, in this definition, includes maintaining a healthy suitable environment to prevent the further spread of disease, such as having clean water and food sources and minimizing potential zoonotic vectors; in short, one all-inclusive (One Health) free or affordable health care system [7].

If Nepal is to move forward in its battle against rabies, programs must be developed that can act within the social, economic and religious ecology of each district and tackle both vaccinations and population control. Additionally, more policy-relevant research must be conducted on the ecology of stray dog populations across the country and the contributions of the sylvatic cycle in order to develop realistic, long-term control programs.

Rabies provides the best zoonotic example of how animals, particularly stray dogs, can affect local economies because it is one of few diseases that are $100 \%$ fatal if left untreated. Additionally, the incubation period is only a few hours and a human or animal can die within days of getting bit. Instances of rabies also show the greatest disparity between industrialized and agrarian nations, where it is all but eradicated in countries like the United States and England, and 2,700 people still die from it each year in Ethiopia. More than $95 \%$ of human deaths caused by rabies occur in Africa and Asia alone [8]. Because of its zoonotic capacity, rabies has a large impact on public health and can greatly impact the economic wellbeing of a community. A study conducted by the veterinary medical school in Ethiopia analyzed the economic impact of rabies in developing countries, both on human health care and livestock mortality, in an attempt to show why it is still a significant threat to transitioning countries. GemechuRegea used data from the Ethiopian Disease Control databases, the veterinary school and comparative studies in Asia and Latin America. He also used a cost benefit analysis to determine the overall economic impact of Rabies in Ethiopia. During the course of the study it was found that rabies transmitted by dogs is responsible for the loss of over 1.8 million DALYs (disability adjusted life years) per year, with direct and indirect economic costs (PEP, animal tests, dog vaccination, and livestock losses) totaling $\$ 5.5$ billion. With the inclusion of costs associated with the risk of human mortality, the global cost for canine rabies becomes $\$ 120$ billion [8]. Whether or not rabies is recognized all over the world, it is still having a substantial impact on the global economy and even more so in the areas where rabies is more heavily concentrated-Africa and Asia.

In further examination of the rabies epidemic in Ethiopia through Regea's study [8], in Tanzania and Kenya with [9] [10]. It is clear that there several outlets through which rabies can affect personal and community economics. One such outlet is through livestock and working animal mortality. Livestock play a large role in agrarian societies like Ethiopia, India or Nepal where they act as a source of income, food, transportation or more. If infected with rabies, the animal is likely to die but before then may infect other animals in the herd, the owners, or may even be sold or slaughtered while infected, further impacting food and economic security. In addition, paying for transport and the expensive postexposure prophylaxis for exposed family or community members can pose significant economic restraint, if it can be afforded at all. Loss of income while seeking PEP, and death in unsuccessful cases, reduce consumer spending throughout the economy. Livestock death also reduces producers' incomes, resulting in a fall in spending in other sectors of the local and regional economy [8]. These macroeconomic effects show how rabies, and other diseases brought on by stray animal overpopulation can affect members of the community who are not in direct contact with the issue.

With the recent urban expansion in both Ethiopia and Nepal, livestock and stray dogs are more likely to come in contact with rabies infected wildlife (foxes, bats, monkeys) drawn in by the smells of the city. Stray dogs then make the perfect vector for the transfer of rabies into urban and peri-urban areas, affecting livestock, humans and companion animals. Moreover, countries with a large tourism industry, such as Nepal, can also be drastically affected by rabies outbreaks and advisories reducing the number of tourists. The physical and psychological tolls incurred from dealing with rabies cases in humans or animals can reduce productivity in those communities, households, and hospitals managing the cases.

It is obvious that implementing One Health programs that may reduce instances of human rabies through the reduction of canine rabies (namely through vaccine programs or combined vaccine and sterilization programs), can be more cost effective than solely preventive treatment with post-exposure-prophylaxis (PEP)-the system that currently exists in Nepal. The study in Ethiopia by [8] commends vaccination programs as the most 
cost-effective way to eliminate rabies as compared to the current economic strain produced by the disease.

\section{Conclusion}

Our world embodies a high level of interconnectedness. As countries industrialize and push through technological revolutions, this creates the misconception that the connections between various classes of human society as well as between humans and animals are trivial or can be easily compartmentalized. However, evidence clearly shows that these connections are so intertwined that, in the area of health care, one diseased stray animal or impoverished human can start an epidemic that may impact millions. An allinclusive global health care approach is sorely needed.

\section{References}

1. World Health Organization, 2007. Nepal health situation reports

2. Heritage Foundation. 2017. "Nepal". Index of Economic Freedom. retrieved from https://www.heritage.org/index/country/nepal

3. Panthhe PK, McCutcheon LA. Rural Urban Education in Nepal. Journal of International Economics. 2015; 96(1): 30-44.

4. Devleesschauwer B, Aryal A, Sharma BK, et al. Epidemiology, Impact and Control of Rabies in Nepal: A Systematic Review. PLoS Neglected Tropical Diseases. 2016; 10(2): 44-61. doi: 10.1371/journal.pntd.0004461

5. Devkota RS. Socio-economic Development in Nepal: Past Mistakes and Future Possibilities. South Asia Economic Journal. 2007; 8(2): 285-315. doi: $10.1177 / 139156140700800206$

6. Miller M. The Threats Humans Present to Wildlife through Infectious Diseases. The Conversation-Environment and Energy. 2015; 4-6.

7. Berrian AM, Smith MH, Van Rooyen J, et al. A Community-based One Health Education Program for Disease Risk Mitigation at the HumanAnimal Interface. One Health. 2018; 5(1): 9-20. doi: 10.1016/j. onehlt.2017.11.002

8. Regea G. Review on Economic Importance's of Rabies in Developing Countries and Its Controls. Archives of Preventative Medicine. 2017; 2(1): 015-021.

9. Stockstad E. Taming Rabies. Science. 2017; 355(6322): 238-242. doi: 10.1126/science.355.6322.238

10. Fitzpatrick MC, Shah HA, Pandey A, et al. One Health Approach to CostEffective Rabies Control in India. Proc Natl Acad Sci U S A. 2016; 113(51): 14574-14581. doi: 10.1073/pnas. 1604975113 